\title{
Prevalence of oral premalignant and malignant Lesions among referred Kurdish patients Attending Department of Oral and Maxilofasial in Sulaimani Teaching Hospital
}

\author{
Assistant Prof. Dr. Shanaz Mohammad Gaphor, Dr. Zhwan Abdulla Sabri
}

\begin{abstract}
Background: oral premalignant and malignant prevalence studies are important to know the state of health and the need of treatment. Premalignancy include group of diseases that acts as precursors of the squamous cell carcinoma. They are clinically and histologically distinct lesions preceding malignant changes. Oral squamous cell carcinoma is a common malignancy that has high metastasis potential and thus survival rate of patients is low.

Aim of study: to investigate the prevalence of oral precancerous and cancerous mucosal lesions among 1000 Kurdish referred patient attending Department of Oral

And Maxillofacial Surgery in Sulaimani Teaching Hospital.

Materials and methods: This cross sectional study was performed on 1000consecutive outpatients, who referred to Sulaimani Teaching Hospital, Department of Oral and Maxillofacial, from November 2012-June 2013. A structured questionnaire was used to collect information which was completed by eachpatient. Biopsy was taken for oral premalignant and malignant lesions. Data were analyzed using the Statistical Package for Social Science (Version 16). Cross tabulation and Chi-square with Fisher's exact test were used.

Result: Among the 1000 patients, 23 patients (2.3\%) had oral premalignant and malignant lesions. The proportion of lesions with potential to transform into cancer was (1.5\%). Sqaumous cell carcinoma observed in $0.8 \%$. Males constituted $43.4 \%$

$(n=10)$ and females $56.6 \%(n=13)$. Lichen planus were the most commonly observed lesions $(n=6,0.6 \%)$ followed by chronic candidial leukoplakia $(n=5,0.5 \%)$, leukoplakia $(n=3,0.3 \%)$ and nicotinic stomatitis $(n=1$, $0.1 \%)$.All lesions had female propensity (56.6\%).The most common location for oral premalignant lesions was buccal тисоsa (41.1\%) followed equally by tongue and palate (23.5\%), but in oral malignant lesions (sqoumous cell carcinoma) tongue was most frequently involved site (37.5\%).

Conclusion: The study has provided information about prevalence of oral premalignant and malignant lesions that may prove valuable in planning of future oral health status.

Key words: prevalence, malignant and premalignant oral lesions.
\end{abstract}

\section{Introduction}

Oral carcinoma is a global health problem with rising prevalence and mortality rates. Oral cancer is the eighth most common cancer worldwide (Kunjan et al., 2005; Peterson, 2005). Oral squamous cell carcinoma is the most frequent of oral carcinomas and represents approximately $3 \%$ of all human malignant tumors (Brandizzi et al., 2008). SCC represents more than $90 \%$ of all head and neck cancers. Is the most common malignant tumor of the lip and oral cavity (90\% of the cases), while the remaining $10 \%$ of the cases are mainly melanomas, sarcomas, minor salivary gland carcinomas and metastatic cancers (Scully, 2006). Premalignant or precancerous lesion is defined by the WHO (1978) as "a morphologically altered tissue which cancer is more likely to occur than its normal counterpart (Warankulasuriya et al., 2007).A premalignant condition is a generalized state associated with a significantly increased risk of cancer (Goyal etal. 2012).A potentially malignant disorder is a lesion or condition, in which risk of malignancy, being present at the time of initial diagnosis or at a future date (Goyal etal, 2012). Oral premalignant lesions are relatively common, occurring in about $2.5 \%$ of the general population and are an important target for cancer prevention (Huber, 2006).Different lesions have been reported to have potential to transform into SCC. Among these, the most frequently mentioned are leukoplakia, lichen planus, erythroplakia, chronic hyperplasic candidial infection, oral sub mucous fibrosis and nicotine stomatitis. The study designed to investigate the prevalence of oral precancerous and cancerous mucosal lesions among 1000 Kurdish referred outpatient to obtain pilot data useful in planning an oral health database for the country.

\section{Material and methods:}

Cross sectional study included 1000 consecutive Kurdish outpatients who referred to Sulaimani Teaching Hospital, Department of Oral and Maxillofacial from November 2012- June 2013.Patient consisted of 
(539) females(53.9\%) and (461) males (46.1\%), age range between (10-69) years. Confidentiality of the patients was considered, the ethical agreement is been made with Sulaimani Teaching Hospital. An interview was conducted to collect information using a structured questionnaire which was completed by each patient and the examiner. Participants after receiving detailed information about the procedure, asked them about socio demographic characteristics, chief complain, date and duration of lesions, filled out a precoded questionnaire about tobacco and alcohol use, dietary habits, and denture wearing. Both dental and general medical histories of patients were obtained. A completed medical history was obtained including history of previous hospitalization, pregnancies, allergy to food or drugs, present medications, and history of systemic diseases. During the clinical examination, the following elements including features of the lesion, anatomical location, extension, etiological factors were recorded. The examination of the oral mucosa and soft tissues in and around the mouth was carrying out following the WHO (1997) criteria, which is thorough and systemic .Diagnosis was established by history of present illness, clinical examination and biopsy that was taken from all premalignant and malignant lesions.

Statistically analysis:

SPSS version16 (Statistical Package for the Social Sciences) (SSPSInc. Chicago, IL) was used .Comparisons were then carried out using Pearson chi-square test (fisher exact test was used if expected frequency is less than 5). $\mathrm{p}<0.05$ regarded as statistically significant, $\mathrm{p}<0.01,0.001$ regarded as highly significant, and $\mathrm{p}>0.05$ regarded as insignificant.

\section{Results:}

The sample consisted of 1000 patients. Males constituted $46.1 \%(\mathrm{n}=461)$ and females $53.9 \%(\mathrm{n}=539)$. Their age ranged between (10-69) years, mean age (34.7) years. The largest number of patients between age group (30-39) $(n=250,25 \%)$, males $(n=103,22.3 \%)$, females $(n=147,27.3 \%)$ and the smallest number of patients between age group $(10-19)(n=86,8.6 \%)$, males $(n=39,8.5 \%)$, females

$(\mathrm{n}=47,8.7 \%)$.

\section{Frequency distributions of oral premalignant and malignant lesions in relation to sex and age}

Table 1 shows the distributions of oral premalignant lesions prevalence according to patients' sex. Among 1000 patients 23(2.3\%) patients had oral premalignant and malignant lesions. Oral premalignant lesions were present in $1.5 \%(\mathrm{n}=15)$ of the total sample. The most common oral premalignant lesions were lichen planus. It was observed in $(0.6 \%)$ of all patients as shown in table (1). All detected OLPlesions were erosive type. It was more prevalent among females $0.7 \%$ than in males $0.4 \%$ but the difference was not statistically significant. The age range of patients with lichen planus was between (20-69) years, the most commonly affected age group was between (40-49) years as shown in table(1). Oral candidial leukoplakia (CL) was seen in $(0.5 \%)$. It was significantly more common among females $(0.9 \%)$. The age range of patients was between (50$69)$ years, the most commonly affected age group was between (50-59) years. Oral leukoplakia was observed in $(0.3 \%)$ of the studied population. It was more prevalent in males $(0.4 \%)$, than females $(0.2 \%)$, but difference was not statistically significant. The age range of patients was between (60-69) years; which was the most commonly affected age group. Nicotinic stomatitis was observed only in one male patient $(0.1 \%)$ at age 65 years old.

Among the 1000 patients, $8(0.8 \%)$ patients had oral SCC. It was more prevalent among males $1.3 \%$ than in females $0.4 \%$ but difference was not statistically significant. The age range of the patients with SCC was between (50-69) years, the most commonly affected age group was between (60-69) years.

Table 1 Distribution of oral premalignant and malignant lesions according to age and sex

\begin{tabular}{|c|c|c|c|c|c|c|c|c|c|}
\hline & & \multicolumn{5}{|c|}{ Diseases } & \multicolumn{2}{|c|}{ Total } & \multirow[t]{2}{*}{ P. value } \\
\hline & & LP & CHC & OL & NS & SCC & No. & $\%$ & \\
\hline \multirow{2}{*}{ Sex } & Female & 4 & 5 & 1 & 1 & 2 & 13 & 6.6 & \multirow{2}{*}{0.08} \\
\hline & Male & 2 & 0 & 2 & 0 & 6 & 10 & 43.4 & \\
\hline \multirow{6}{*}{ Age } & $10-19$ & 0 & 0 & 0 & 0 & 0 & 0 & 0 & \multirow{6}{*}{0.23} \\
\hline & $20-29$ & 1 & 0 & 0 & 0 & 0 & 1 & 4.3 & \\
\hline & $30-39$ & 1 & 0 & 0 & 0 & 0 & 1 & 4.3 & \\
\hline & $40-49$ & 4 & 0 & 0 & 0 & 0 & 4 & 17.3 & \\
\hline & $50-59$ & 0 & 3 & 0 & 0 & 3 & 6 & 26.08 & \\
\hline & $60-69$ & 0 & 2 & 3 & 1 & 5 & 11 & 47.8 & \\
\hline
\end{tabular}

Pearson chi-square test was used, $\mathrm{p}<0.05$ regarded as significant. 


\section{Site distribution of oral premalignant and malignant lesions}

On the basis of site Involvement; in the oral premalignant lesions, buccal mucosa was found to be most frequently involved site(41.1\%), followed equally by tongue and palate (23.5\%), figure (1)shows the site distribution of oral premalignant lesions. OLP localized in buccal mucosa in 6 patients (75\%), followed equally by tongue and gingival area (12.5\%). Oral $\mathrm{CHC}$ which localized in palate in 3 patients $(60 \%)$, and followed by tongue in 2 patients (40\%). OL which distributed equally on buccal mucosa, tongue and alveolar ridge $(33.3 \%)$. Nicotinic stomatitis was, located at the palate (100\%). Most common site of squamous cell carcinoma was tongue $(37.5 \%)$ followed equally by buccal mucosa and floor of mouth $(25 \%)$ and lip $(12.5 \%)$.

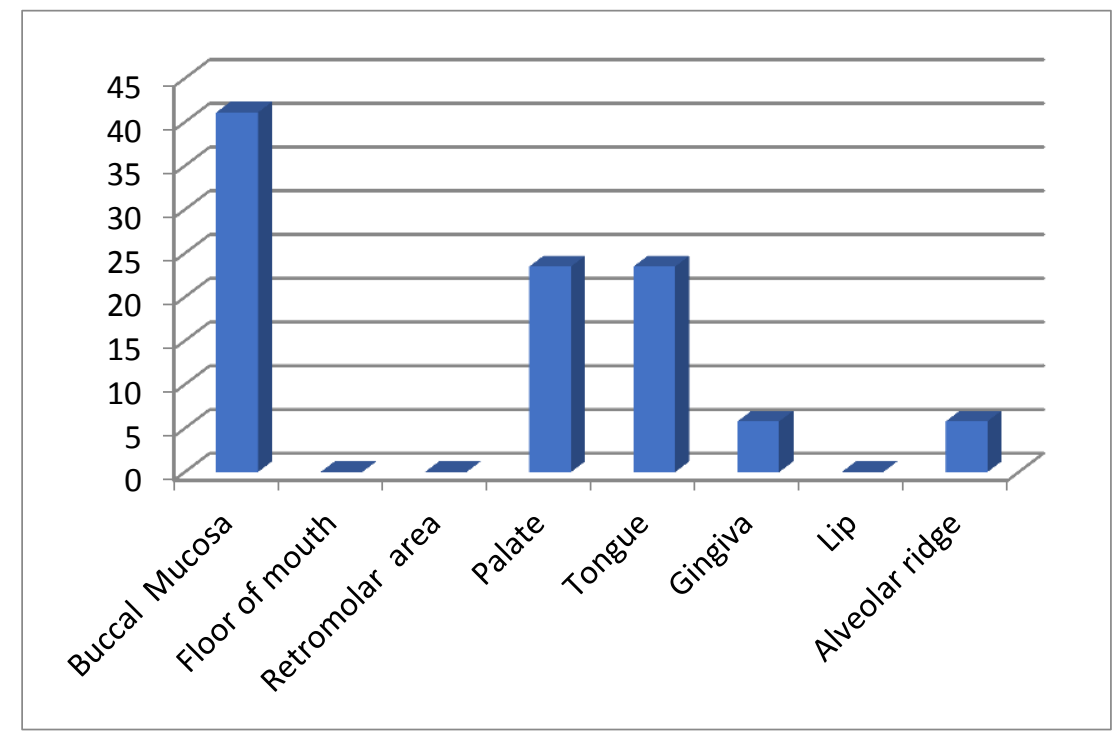

Figure (1) Site distribution of oral premalignant lesions

\section{Discussion:}

Today, cancer is one of the leading threats to human life. Studies on precancerous lesions are very important since it is known that oral cancers still cannot be diagnosed adequately in early stages. The role of the dentist in detecting oral carcinomas and premalignant lesions is crucial. The main way to deal with this problem is to educate people about the importance of regular dental checkups and the relationship that many of the associated factors have with the development of oral premalignancy and malignancy (Goyal et al., 2012). Correct diagnosis and timely treatment of potentially malignant lesions with high risk of malignant transformation may help to prevent malignant transformation. Potentially malignant lesions can be treated based on the clinical and histological staging. Various treatment modalities are suggested in the literature; from cessation of the habit to surgical reconstruction. But among this early diagnosis of the lesion and cessation of habit is the best line of treatment (Hakan et al., 2003).

Prevalence studies in dentistry are mostly based on either the examination of total population samples or dental outpatients. The study made on patients referred to the Sulaimani Teaching Hospital, Oral and Maxillofacial Department. Among 1000 patients only 23 patients (2.3\%) had oral premalignant and malignant lesions, a result comparable with that found in a study by Saraswathi et al.(2006) in Chennai ( 4.1\%), and very low to that found by Mehrotra et al (2010) in India (67\%), and Swedish study that were done by Axell(1987) $(13.8 \%)$.

These variations could be explained due to:

1- Age and sex distribution of the sample.

2- Different methodologies used.

3- Specific cultural habits like smoking and use of alcohol.

4- Geographical, racial, and socioeconomic factors.

5- Educational level and cultural levels of patients.

6- Use of dentures.

7- Systemic diseases and medication used.

8- Food type.

The male to female ratio reported in this study was 1:3. This is disagreement with the finding of Hakan et al. (2003) in Turkey in which premalignant and malignant lesions were more prevalent among males than in females. This could be due to that females are more seek medical attention or it could be females more equally exposing themselves to associated risk factors .squamous cell carcinoma more common among males 
$(1.3 \%)$.However the difference in the ratio is not significant. The number of patient in the different age groups demonstrated that these lesions are more commonly among elderly patients. The age related suggested that time dependent factors result in initiation and promotion of cellular changes that results in malignant change.

OLP is ranked first most common oral premalignant lesions. This finding agrees with study done by Hakan et al.(2003) in Turkey but disagreement with studied done by Mehrotra et al.(2010)in India and Zain et al(1997) in elderly Malaysians.

OLP, was present in $(0.6 \%)$ of the total sample. This is comparable to that found among Swedish people (1.9\%) Axell and Rundquist (1987), and; Axell et al. (1990) reported rates of 3.8\% and $2.1 \%$ in Thai and Malaysian outpatients respectively. The worldwide prevalence of the OLP in the general population has been estimated to range from $0.1 \%$ to $2.2 \%$ (Jungell, 1991).We confirm that OLP is more frequent in females $(1.1 \%)$ than in males $(0.4 \%)$. The most affected group was $40-49$, this is compatible with other studies that were done by Anbar et al. (2005) among Egyptians Al-Minya province, and Pakfetrat et al.(2009) in which OLP was more common among females, because stress was most common among females which is most common etiological factors of lichen planus (Ivanoviski et al.,2005).

The prevalence of oral candidial leukoplakia in our study was $(0.5 \%)$ and all lesions observed in females $(0.9 \%)$ and in older age group(50-60), because denture wearing more common at this age group . This is comparable to the finding by Ikeda et al. (1995) (1.4\%) and more than that found by Alxell et al. (1990) in Kuala Lumpur $(0.4 \%)$.

Leukoplakia was seen in (0.3\%), it is compatible to the finding by Shulman et al (2004) in USA adult( $(0.3 \%)$, but low when compared with the studies by Ikeda in Japan (25\%), Rooban et al.(2009) in Chennai, South India(7.4\%) and Espinoza et al.(2003) in Santiago, Chile, (1.7\%).These large variations may be related to the diagnostic criteria used to classify cases and different demographic composition of the study populations. All cases of leukoplakia seen at ages 60 and older. It was more prevalent in male than female $(0.4 \%$ and $0.2 \%)$; however, sex difference was not statistically significant. This is probably because we have few cases of leukoplakia (only 3cases) which is agreement with the finding of Lpthanasupkul et al. (2007) in Thai population.

Nicotinic Stomatitis was observed only in one male $(0.1 \%)$, the prevalence was lower than that observed in AL mobeeriek and Aldosari (2009) in Saudi Arabia (0.51\%) and in Shulman et al. (2004) in USA adults $(0.50 \%)$.

Squamous cell carcinoma was seen in $0.8 \%$. Males $(1.3 \%)$ had a higher predilection for this lesion than females $(0.4 \%)$, it was observed more often in age group of (50-60) years .This prevalence is more than that found by Cebeci et al.(2009) in adult Turkish population (0.06\%), Zain et al. (1997) in Malaysia(0.04\%), and lower than that found by Jahanbani et al (2009) in Tahran (1.7\%).

Detection of locations of oral premalignant and malignant lesions was important because prognosis of oral cancer differs significantly between specific oral locations. The buccal mucosa was most frequently involved site among oral premalignant lesions; however tongue was more frequent in SCC, followed by buccal mucosa. It was in agreement with a study done by Mehrotra et al (2010) at Tertiary level Hospital in Allahabad, India, in which buccal mucosa was most common site involved among oral pre malignant lesions (47.7\%), tongue was most frequent involved site among oral malignant lesions (67.4\%). While the study was disagreement to that found by Shulman (2005) in the USA, where lip was most frequent site of lesions. The majority of the OLP were seen in the buccal mucosa, followed equally by tongue and anterior gingival. This was in agreement with other studies done by Xue et al. (2005) in China, Pakfetratet al. (2009) among Iranian patients and Oliveria et al. (2010) among Brazilian patients, in which cheek mucosa was the most site affected, followed by the tongue and gingival.

In squamous cell carcinoma, tongue was the first frequent site followed by buccal mucosa because tongue is one of these areas of mouth that covered by a thinner, non-keratinized mucosa which provides less protections against carcinogens, this result was similar to Iype et al. (2001) in Trivandrum who reported 52\% of their patient had tongue involvement followed by buccal mucosa(26\%), and Jahanbani et al(2009) in Tehran (53\%), while(Bhurgri,2005) suggested in her reported from South Karachi, Pakistan that ,buccal mucosa was the most frequently involvement site (55.9\%), followed by tongue(28.4\%).The reason for the variation in the distribution of affected oral locations is may be because of different methodologies used, the number and type of lesions included in the studies and habits (life styles).

\section{Limitation of the study}

There are few studies reporting oral premalignant and malignant lesions in general population due to the difficulties of the method .Examination of dental out patients is easier to perform but it does not give information about the whole population. Interpretation of data from a single institution has clear limitation. The data reflects the specific patient population reporting to the Sulaimani Teaching Hospital and not the community as a whole and excluding non-Kurdish people. 


\section{Conclusion:}

The result of this study provides some information on the prevalence of oral premalignant and malignant lesions in referred Kurdish outpatient. Potentially malignant disorders are an important spectrum of diseases that need to be identified and followed up closely. Correct identification of their malignant potential may help in early diagnosis of cancer and down staging of the diseases.

\section{References:}

[1]. AL-Mobeeriek A, Aldosari AM (2009).Prevalence of oral lesions amongSaudi dental patients.Ann Saudi Med; 29:365-368

[2]. Anbar Tag-EI-Din, BarakatM,Ghannam SF(2005).A clinical andepidemiological study of lichen planus among Egyptians of ALMinyaprovince. Dermatol Online J; 11:50

[3]. Axell T, Rundquist L (1987). Oral lichen planus. A demographic study.Community Dent Oral Epidemiol 15: 52-6.

[4]. Axell T, Zain RB, Siwamogstham P, Tantiniran D, Thampipit J(1990).Prevalence of oral soft tissue lesions in out-patients at two Malaysian andThai dental schools. Community Dent Oral Epidemiol 18: 95-9.

[5]. Axéll, T. (1987), Occurrence of leukoplakia and some other oral whitelesions among 20333 adult Swedish people. Community Dentistry and OralEpidemiology, 15: 46-51.

[6]. BhurgriY (2005).Cancer of oral cavity-trends in Karachi SouthAsian Pac JCancer Perv; 6,420.

[7]. Brandizzi D, Gandolfo M, Velazco M L, Cabrini R L, Lanfranchi H E(2008). Clinical features and evolution of oral cancer: A study of 274 casesin Buenos Aires, Argentina. Med Oral Patol Oral Cir Bucal. Sep1; 13:E544-8.

[8]. Cebeci AR I,GulsahiA,KamburogluK,OrhanBK,OztasB(2009).Prevalenceand distribution of oral mucosal lesions in an adult Turkish population.MedOral Patol Oral Cir Bucal.Jun 1:14; E272-7.

[9]. Espinoza I,RojasR,ArandaW,Gamonal J(2003).Prevalence of oral mucosallesions in elderly people in Santiago,Chile.J Oral Pathol Med;32:571-5.

[10]. GoyalD,GoyalP,SingHP,Verma(2012).Precancerous lesions of oral cavity.OralSurg Oral Med Oral Pathol: IJMDS;2:70-71.

[11]. HakanAkman,EnisPedzep,Umit K(2003).Prevalence of oral premalignantlesions in a selected Turkish population,TurkJ,Med Sci;33:39-42.

[12]. Huber MA (2006). A review of premalignant oral conditions. Tex Dent J;123:502-509. Ikeda N,HandaY,KhimSP,DarwardC,AxellT,Mizuna T(1995). Prevalence study of oral mucosal lesions in a selected Cambodianpopulation.Community Dent Oral Epidemiol; 23:49-54.

[13]. IypeEM,PandyM,Mathew A (2001).Oral cancer among under the age of 35years.J Postgrad Med,47:171-6.

[14]. Ivanovski K, Nakova M, Warurton G, Pesevska S, Filipovska A, Nares S,Nunn ME, Angelova D, Angelov N (2005). Psychological profile in orallichen planus. J ClinPeriodontol; 32:1034-1040.

[15]. Jahanbani J, Sandvik L, Lyberg T, Ahlfors E (2009). Evaluation of oralmucosal lesions in 598 referred Iranian patients. The Open Dent J; 3: 42-47.

[16]. Jungell P (1991). Oral lichen planus .A review.Int J Oral MaxillofacSurg;20:129-35.

[17]. Kunjan O, Glenny AM, Duxbury AJ (2005). Evaluation of screeningstrategies for improving oral cancer mortality: a cochrane systematic review.J Dent Edu, 69, 255-66.

[18]. LapthanasupkulP,PoomsawatS,Punyasingh J(2007).A clinicopathologicstudy of oral leukoplakia and erythroplakia in aThaipopulation.Quintessence Int;38:16:20.

[19]. Mehrotra R, Thomas S,NairP,PandyaS,SinghM,NigamNS,ShuklaP(2010).Prevalence of oral soft tissue lesions in Vidisha .BMC ResNotes.25;3-23.

[20]. Oliveria Alves, MonicaGhislaine,AlmeidaJanete,BalducciIvan,CabralLuiz, Antonio Guimaraes(2010).Oral lichen planus :A retrospective study of110 Barazillianpatients.BMC Res Notes;3:157.

[21]. PakfetratAtessa,Javadzadeh-BolouriAbbas,Basir-Shabestari Samira, Falaki

[22]. Farnaz(2009). Oral lichen planus:A retrospective study of 420 Iranianpatients.Med Oral Patol Oral Cir Bucal.1;14;E315-8.

[23]. Peterson PK (2005). Strengthening the prevention of oral cancer: the WHOperspective. Community Dent Oral Epidemiol, 33, 3979.

[24]. RoobanT,RaoA,JoshuaE,Ranganathan K(2009). The prevalence of oralmucosal lesions in alcohol misusers in Chennai,South India.Indian J DentRes;20:41-6.

[25]. SarawathiTR,RanganathanK,ShanmugamS,SowmyaR,NarasimhanPD.Gunaseelan R(2006).Prevalence of oral lesions in relation tohabits:Cross sectional study in South India.Indian J Dent Res;17:121-5.

[26]. Scully C (2006). Clinical practice. Aphthous ulceration. N Eng J Med.13;355: 162-72.

[27]. Shulman JD (2005).Prevalence of oral mucosal lesions in children and youthin the USA.Int J Paediater Dent; 15:89-97.

[28]. Shulman JD, Beach MM, Rivera-Hidalgo F (2004). The prevalence of oralmucosal lesions in U.S. adults data from the Third National Health andNutrition Examination Survey, J Am Dent Assoc; 135: 1279-1286.

[29]. Warnakulasuriya S, Johnson NW, Vanderwaal (2007). Nomenclature andclassification of potentially malignant disorders of the oral mucosa J OralPathol Med; 36:575-80

[30]. World Health Organization Collaborating Centre for Oral Precancerouslesions (1978) Definition of leukoplakia and related lesions: an aid to studieson oral precancer. Oral Surg Oral Med Oral Pathol; 46: 518-39.

[31]. Xue JL,FanMW,WangSZ,ChenXM,LiY,Wang L(2005).Aclinical study of674 patients with oral lichen planus in China,J Oral Pathol Med ;34:467-72.

[32]. Zain RB,IkedaN,RazakIA,AxellT,MajidZA,GuptaPC,Yaacob M(1997).Anational epidemiological survey of oral mucosal lesions in Malaysia,Community Dent Oral Epidemiol.Oct;25(5):377-83 